\title{
CHRONIC EOSINOPHILIC LEUKAEMIA PRESENTING WITH A CARDIAC MASS: A CASE REPORT
}

AHMEDUL KABIR ${ }^{1}$, MD. ROBED AMIN ${ }^{2}$, PRATYAY HASAN ${ }^{3}$, SHEGUFTA MISHKET MUKERRAMA ${ }^{4}$, MUHAMMAD NURUL FARHAD ${ }^{5}$, SUDIP RANJAN DEB ${ }^{6}$, ANWAR HOSSAIN ${ }^{7}$

\begin{abstract}
Chronic eosinophilic leukaemia is a rare disorder. It can cause irreversible target organ damage which can be life threatening,so early diagnosis of the disease is a necessity.It may pose as a diagnostic dilemma. Active consideration of the possibility of the disease is necessary. We intend to report a case of a boy of 13 years who presented with hepato-splenomegaly, generalized lymphadenopathy, a cardiac mass and a very high eosinophil count. He was ultimately diagnosed with chronic eosinophilic leukaemia.Though rare, this disease can occur in our country, too. Unavailability of confirmatory tests warrants more vigilance and awareness among physicians.
\end{abstract}

\section{Introduction}

Chronic eosinophilic leukaemia is a rare blood disorder. Its current true incidence or prevalence is not well established. In our country it is even less familiar. Recent advancement of methods of investigation and identification of previously unknown genetic abnormalities has elucidated much on the disease process, but those methods and techniques are yet not widely available. This disease involves various vital organs in the body, causes irreversible target organ damage and may pose as a diagnostic dilemma owing to its wide variety of manifestations. Some of its manifestations may be overlooked or neglected causing delay or misdiagnosis which might result in significant amount of mortality and morbidity. We want to report a case of a boy of 13 years with chronic eosinophilic leukaemia that affected various systems with bizarre presentation.

\section{Case Report}

Shakil, 13 years, Muslim boy, hailing from North Badda, Dhaka, presented with history of fever for 1 and half months which was high grade, but not associated with chills and rigors, appeared in the evening and usually remitted before night, often with sweating. But later he remained fever-free for only 34 hours in a day. He had no history of cough, hemoptysis, respiratory distress, gum bleeding or bleeding from any site of the body, bone pain, sore throat, head-ache, vomiting, weakness of limbs, alteration of bowel and bladder habit.He had no significant past history, associated illness or family history.On examination, he was found mildly anaemic, pulse $88 / \mathrm{min}$, BP 90/60 mm-Hg, with multiple lymph nodes in the anterior and posterior cervical chains, submandibular and sub lingual regions bilaterally, and also in right groin. The lymph nodes were small in size, firm in consistency, non-tender, not fixed with overlying skin, or underlying tissue and no draining sinus were present. The biggest one measured about $1.5 \mathrm{~cm}$ in diametre. $1 \mathrm{st}$ and $2 \mathrm{nd}$ heart sounds are audible and normal.Pericardial rub was absent.A pan systolic murmur was heard which was loudest at the apex but radiated widely over the precordium and into the axilla, best heard with diaphragm of the stethoscope in supine position with breath held in expiration.

1. Associate Professor, Department of Medicine, Dhaka Medical College\& Hospital, Dhaka

2. Associate Professor, Department of Medicine, Dhaka Medical College Hospital, Dhaka

3. Medical Officer, Department of Medicine, Dhaka Medical College \& Hospital, Dhaka

4. Medical Officer, Department of Medicine, Dhaka Medical College \& Hospital, Dhaka

5. Medical Officer, Department of Haematology, Dhaka Medical College \& Hospital, Dhaka

6. Assistant Professor, Department of Medicine, Dhaka Medical College \& Hospital, Dhaka

7. Assistant Registrar, Department of Medicine, Dhaka Medical College \& Hospital, Dhaka

Address of Correspondence: Dr. Ahmedul Kabir, Associate Professor, Department of Medicine, Dhaka Medical College \& Hospital, Dhaka

Bangladesh J Medicine 2014; 25 : 25-28 
Later he complained about mild respiratory distress, and his chest auscultation revealed bilateral basal crepitation.Examination of other systems revealed no abnormality.

As for investigations, a chest X-ray was done, which showed Slightly full pulmonary conus, otherwise normal chest x-ray. A CBC showed HGB $9.4 \mathrm{~g} / \mathrm{dL}$, ESR $82 \mathrm{~mm}$ at $1 \mathrm{st}$ hour, RBC 3.2 million/cu mm, WBC 20,000/cu mm, CE 10,000/cu mm. [Polymorphs 24\%, Lymphocytes 20\%, Eosinophils 50\%, Monocytes 6\%]. An USG of whole abdomen revealed mild hepatomegaly with homogenous echo-pattern and mild splenomegaly $(12.6 \mathrm{~cm})$ with uniform echotexture., A PBF showed Hypochromic microcytic anaemia with gross eosinophilia. Pencil form cells and few target cells were seen. Blood Culture revealed no growth at 37 degree Celsius for 72 hours. An echocardiography showed intra-cardiac mass involving LV and RV, MR grade III and TR grade II, mild pericardial effusion, good LV function (EF:58\%). ICT for kala-azar and malaria were negative. Findings of serum iron profile were as such: ferritin : $169.11 \mu \mathrm{g} /$ $\mathrm{L}$, serum total iron was $61 \mu \mathrm{g} / \mathrm{dL}$ TIBC was $215 \mu \mathrm{g} / \mathrm{dL}$ (normal: 250-425 $\mu \mathrm{g} / \mathrm{dL}$ ). A repeat chest x-ray showed Atrial enlargement, straightening of the left heart border, globular heart shadow. A repeat CBC after 23 days of admission showed normocytic anaemia with eosinophiliaWBC: 22.63 X 103/ $\mu \mathrm{L}$ (neutrophil 45\%, eosinophil 40\%, lymphocyte 15\%, monocyte 5\%)RBC: 2.16 X $106 / \mu L H b: 6.7$ g/dLHct: $21.7 \%$ MCV: 98.6 fLAgain a bone marrow biopsy taken from the posterior superior iliac spine showed features suggestive of eosinophilic myeloid hyperplasia and described the findings as hyperactive with left shift maturation and also shows mature eosinophils and significant number of eosinophilic myelocytes. Eosinophils had sparse granulation with clear area of cytoplasm, cytoplasmic vacuolation, nuclear hyper and hyposegmentation. A serum vitamin B-12 assay showed $708.70 \mathrm{pmol} / \mathrm{L}$ and high uric acid. LAP/ NAP score was within normal range. Biopsy from posterior cervical lymph node commented reactive hyperplasia, low grade lymphoma should be ruled out. Description of lymph node biopsy was as follows: Infiltration of subscapular sinus and para-cortical areas by eosinohils and plasma cells, fair number of lymphoid follicles but no prominent germinal centre, many follicles have faded, no malignancy seen.

The patient was put on prednisolone $30 \mathrm{mg}$ at morning and a tyrosine kinase inhibitor: Imatinib $200 \mathrm{mg}$ at morning. The patient was discharged and then followed up after 1 month of admission. He improved symptomatically, not very toxic as he had been before and the complaints of breathlessness were gone.On examination: there were been no hepato-, or splenomegaly found and all his previously palpable lymph nodes regressed in size or were not palpable anymore.

But the patient went home, discontinued treatment 1 month after his admission and then expired due to irreversible cardiorespiratory failure about 2 months after her admission in $\mathrm{DMCH}$.

\section{Discussion}

Eosinophilia may be defined as presence of eosinophils in an amount of greater than $500 / \mu \mathrm{L}$ whereas hypersinophilia means a count greater than $1500 / \mu \mathrm{L}$. Eosinophilia have many causes including allergic reactions (to drugs or certain other antigens), collagen vascular disease, malignancy, chronic granulomatous disease (i.e. TB), etc. ${ }^{1}$ To systematically categorize this array of conditions, we can identify 3 groups $^{2}$ :

Reactive eosinophilia: eosinophils are non-clonal, produced in increased number as a response to an underlying disorder through various mechanisms which ultimately result in increased formation of IL5, IL-3, GM-CSF which in turn raise the eosinophil count $^{2}$. Some examples:

a. Allergy

b. Infection: mostly parasites esp. nematodes and trematodes especially during tissue migration phase, mildly cestodes (unless in case of cyst rupture) and very mildly protozoan infection.

c. Hodgkin's lymphoma

d. T lineage non Hodgkin lymphoma

e. $A L L^{3}$

f. Lymphoproliferative variant of hypereosinophilic syndrome.

2. Clonal eosinophilia: Chronic eosinophilic leukarmia (CEL), systemic mastocytosis (SM), chronic myelomonocytic leukaemia (CMML), acute myeloid leukaemia (AML), myelodysplastic syndrome, etc. In all these conditions eosinophils is part of the neoplastic clone. In CMML, there is also increase in circulating monocytes and in CML circulating granulocyte precursors ${ }^{2}$, but in CEL eosinophils are the predominant cells.

3. Hypereosinophilic syndrome: no cause is found, neither of the above mentioned causations (reactive/clonality) applies to this group. A significant proportion of patients who until quite recently would have been diagnosed as idiopathic hypereosinophilic syndrome are now identified as CEL caused by rearrangement of PDGFRA ${ }^{4}$. 
As is the case of our patient, he presented with mild anaemia, both hepatosplenomegely and generalized lymphadenopathy which were clearly indicative of some undergoing hematological disorder possibly a leukaemic process. Those distinct clinical features together with very high eosinophil count led us to search for reactive causes and clonality. All the reactive causes were excluded first. He was treated with Albendazole twice with an interval of 7 days in between, routine stool examination showed no ova or cyst or parasite and and also investigation for filarial antigen was done which all came negative. He had no history of atopy/ eczema/ allergy or asthma. His respiratory examination revealed no abnormality. His lymph node biopsy showed only reactive hyperplasia and thus excluded any lymphoma. Also there was no atypical lymphoblast present in the blood to indicate ALL or lymphoproliferative variant of hypereosinophilic syndrome. A serum vitamin B-12 assay showed $708.70 \mathrm{pmol} / \mathrm{L}$ which was raised showing further evidence of leukaemic process which caused increased amount of vitamin B-12 receptor ${ }^{2}$ (2), and high uric acid showed evidence of high cell turnover. LAP/ NAP score was within normal range which excluded CML, in which it would have been subnormal ${ }^{5}$. There was no significantly raised level of monocytes so CMML was also excluded. Thus two of the three neoplastic conditions (in which eosinophils are part of neoplastic clone) which can possibly cause such high eosinophil count namely CEL, CMML and CML could be excluded and only CEL remained as the possible diagnosis. Again a bone marrow biopsy taken from the posterior superior iliac spine showed features suggestive of eosinophilic myeloid hyperplasia and described the findings as hyperactive with left shift maturation and also shows mature eosinophils and significant number of eosinophilic myelocytes. Eosinophils had sparse granulation with clear area of cytoplasm, cytoplasmic vacuolation, nuclear hyper and hyposegmentation. These findings are characteristic morphological features of chronic eosinophilic leukaemia ${ }^{6}$. The modern diagnostic criteria proposed by WHO for CEL include $^{7}$ :

1. Persistent eosinophilia $\left(1.5 \times 10^{9} / \mathrm{L}\right.$ in blood with increased marrow eosinophils.

2. $>5 \%$ but $<19 \%$ myeloblasts in bone marrow or $>2 \%$ eosinophils in peripheral blood.

3. Clonality of myeloid cells.

4. No reactive eosinophilia due to allergy orparasitic infection pulmonary or collagen vascular disease.

5. No reactive eosinophilaia due to other malignancies.
6. No T cell population with aberrant population and abnormal phenotype.

Our case meets the morphological criteria of CEL.

Many cytogenetic abnormalities are currently identified as causative factor of CEL. Most common being FIP1-PDGFRA fusion which alone is responsible for $30-60 \%$ of what we previously knew as hypereosinophilic syndrome ${ }^{4}$. Among others are PDGFRB, FGFRA, etc. Though cytogenetic study could not be done, a presumptive diagnosis of eosinophilic leukaemia is justified in view of the markedly high eosinophil count. Besides the characteristic morphology of bone marrow with eosinophilic precursors and dysplastic features confirms the neoplastic nature of eosinophils ${ }^{8}$. So our case falls in category as reported by Chusid ${ }^{9}$ in a series of 14 patients 4 cases were classified as eosinophilic leukaemia depending upon the presence of high eosinophilic leukaemia and immature eosinophilic precursors. 3 of them had splenomegaly.

In our case the cardiac abnormalities can be attributed to persistent hypereosinophilia causing target organ damage. The release of eosinophilic granule contents including eosinophilic cationic proteinand major basic protein may lead to direct endothelial damage causing and/or fibrosis ${ }^{10}$. Cardiac involvement is most common manifestation of target organ damage and may comprise of multiple conditions including mural thrombosis and a unique condition known as Loeffler's endomyocarditis. The latter is characterized by endomyocardial thickening due to fibrosis leading to compromise of the ventricular cavity ${ }^{11}$. In severe diseases the dense fibrotic layer extends to thicken and tether the AV valve leaflets ${ }^{12}$ which results in restricted leaflet motion (restricted opening) type IIIa variety of $\mathrm{MR}^{13}$ Involvement of posterior mitral and tricuspid valve leafletsmay result in $\mathrm{MR}$ and $\mathrm{TR}^{14}$. Cardiac involvement often has biventricular distribution. Cardiotoxiceosinophils (vacuolated and hypogranulated) are central to the pathogenesis ${ }^{14}$.

In addition our patient showed excellent response to imatinib mesylate as evidenced by serial CBCs and indeed imatinib responsiveness helped in identification of many F-P+ (FIP1-PDGFRA positive) CELs $^{15}$.

Our effort is to emphasize the importance of early diagnosis and rapid initiation of treatment as the response to currently available treatment is excellent and delay in treatment may result in irreversible target organ damage with significant mortality and morbidity. 


\section{Conclusion}

Chronic eosinophilic leukaemia is a very rare disease. Owing to its rarity the true incidence and many other aspects of this disease is still unknown. Especially in our country, even in the subcontinent we are not much familiar with the condition. Recent development of new molecular studies has provided excellent chance to diagnose the disease with more specificity and sensitivity but unfortunately we cannot reap its benefit owing to unavailability and non-feasibility of the tests in our country. Hypereosinophilia often may be misinterpreted to be caused only by reactive process like parasitic infection or allergy and the cardiac problems are often maybe misdiagnosed as sequel of rheumatic heart disease. Certainly the other manifestations like hepatosplenomegaly which may be indicative of the underlying ongoing leukaemic process cannot be overlooked or neglected. After all the discussion we are of the opinion that if such clinical problems arise, consideration of CEL should be taken into account and active search for clonality of eosinophils should be undertaken. Proper integration and correlation among the various disciplines is mandatory. Specialists of Internal Medicine may play a crucial, pivotal role in management of such multi system diseases.

\section{References:}

1. Holland S11. Gallin JI. Disorders of granulocytes and monocytes. In Lango DL, Kasper DL, Jameson JL, Fauci AS, Hauser SL, Loscalzo J, editors. Harrison's principles of internal medicine. 18th ed. USA: The Tata-McGraw Hill, Inc.; 2012. p. 481.

2. Fletcher S, 2. Bain BJ. Eosinophilic leukaemia. British Medical Bulletin. 2007;(81 and 82): p. 115-127.

3. Ryan ET, Wilson ME, Kain KC. Illness after international travel. New England Journal Medical. 2002;(347): p. 505-516.

4. Cools J, De Angelo D, Gotlib J. A tyrosine kinase created by fusion of the PDGFRA and FIP1L1 genes as a therapeutic target of imatinib in idiopathic hypersinophilic syndrome. New England Journal of Medicine. 2002;(348): p. 1201-1214.

5. Firkin F, Chesterman C, Penington D, Rush B, editors. de Gruchy's Clinical Haematology in Medical
Practice. 5th ed. London: The Blackwell Science Ltd.; 1989.

6. Rudolf W, Yasemien M, Martin W, Ulrick K, Ulf S, Christian G, et al. Chronic eosinophilic leukaemia (CEL): a distinct myeloproliferative disease. British Journal of Haematology. ;(96): p. 117-123.

7. Vardiman J, Jaffe E, Stein H, editors. World Health Organization Classification of Tumours : Pathology and Genetics of Tumours of Haematopoietic and Lymphoid Tissues Lyon: IARC Press; 2001.

8. B Presentey Z, Mintz U. Eosinophilic leukaemia: morphological, cytochemical and electron microscoope studies. Journal of Clinical Pathology. 1979;(32): p. 261-271.

9. Chusid M, Dale D, West B, Wolff S. The hypereosinophilic syndrome: analysis of fourteen cases with review of the literature. Medicine. 1975;(54): p. 1-27.

10. Weller P, Bubley G. The idiiopathic hypereosinophilic syndrome. Blood. 1994;(83): p. 2759-79.

11. Coutre S, Gotlib J. targeted treatment of hypereosinophilic syndromes and chronic eosinophilic leukaemia with imatinib mesylate. Seminars in Cancer Biology. 2004;(14): p. 23-31.

12. Stevenson L, Loscalzo J. Cardiomyopathy and myocarditis. In Lango DL, Kasper DL, Jameson JL, Fauci AS, Hauser SL, Loscalzo J, editors. Harrison's principles of internal medicine. USA: The TataMcGraw Hill, Inc.; 2012. p. 1967.

13. Adams D, Carabello B, Castillo J. Mitral valve regurgitation. In Fuster V, Walsh R, Harrington R, Hunt R, Prytowsky E, Roberts R, et al., editors. Hurst's The Heart. USA: The Tata-McGraw Hill, Inc.; 2011. p. 1723.

14. Hoit B. Restrictive, obliterative and infiltrative cardiomyopathies. In Fuster V, Walsh R, Harrington R, Hunt R, Prytowsky E, Roberts R, et al., editors. Hurst's The Heart. 13th ed. USA: The Tata-McGraw Hill, Inc.; 2011. p. 873.

15. Gotlib J, Cools JMM, Schrier S, Gilliland D, Coutre S. Practical caveats to the classification of chronic eosinophilic leukaemia and hypereosinophilic syndrome as mutually exclusive diagnoses. Blood. 2004; 104(12). 